\title{
Potent antitumor activity of the Ad5/11 chimeric oncolytic adenovirus combined with interleukin-24 for acute myeloid leukemia via induction of apoptosis
}

\author{
XUBIN WEI ${ }^{1,2}$, LI LIU ${ }^{2}$, GANG WANG ${ }^{2}$, WEI LI ${ }^{2}, \mathrm{KE} \mathrm{XU}^{2}$, HONGYAN QI ${ }^{1}$, \\ HONG LIU $^{1}$, JING SHEN ${ }^{1}$, ZHONGJIE LI ${ }^{1}$ and JIMIN SHAO ${ }^{1}$
}

${ }^{1}$ Department of Pathology and Pathophysiology, Zhejiang University School of Medicine, Hangzhou, Zhejiang 310058;

Received May 7, 2014; Accepted August 12, 2014

DOI: 10.3892/or.2014.3563

\begin{abstract}
The Ad5/11 chimeric oncolytic adenovirus represents a promising new platform for anticancer therapy. Acute myeloid leukemia (AML) is a heterogeneous clonal disorder of hematopoietic progenitor cells and is the most common malignant myeloid disorder in adults. Myeloid and other hematopoietic cell lineages are involved in the process of clonal proliferation and differentiation. In the present study, we aimed to ascertain whether chimeric oncolytic adenovirusmediated transfer of the human interleukin-24 (IL-24) gene induces enhanced antitumor potency. Our results showed that the Ad5/11 chimeric oncolytic adenovirus carrying hIL-24 (AdCN205-11-IL-24) produced high levels of hIL-24 in AML cancer cells, as compared with the Ad5 oncolytic adenovirus expressing hIL-24 (AdCN205-IL-24). AdCN205-11-IL-24 specifically induced a cytotoxic effect on AML cancer cells, but had little or no effect on a normal cell line. AdCN20511-IL-24 induced higher antitumor activity in AML cancer cells by inducing apoptosis in vitro. This study suggests that transfer of IL-24 by an Ad5/11 chimeric oncolytic adenovirus may be a potent antitumor approach for AML cancer therapy.
\end{abstract}

\section{Introduction}

Acute myeloid leukemia (AML) is characterized by the accumulation of immature myeloid cells in the bone marrow and the suppression of normal hematopoiesis. Increased understanding of the pathogenesis of AML has fostered the development of targeted therapies intended particularly for older patients. Successful targeting of each of the numerous

Correspondence to: Dr Jimin Shao, Department of Pathology and Pathophysiology, Zhejiang University School of Medicine, Hangzhou, Zhejiang 310058, P.R China

E-mail: shaojimin@zju.edu.cn

Key words: acute myeloid leukemia, chimeric oncolytic adenovirus, gene therapy, IL-24, apoptosis genotypic variants of AML is a major challenge $(1,2)$. AML blasts develop from normal blasts affected by two types of genetic damage. The first (class 1) results in constitutive activation of cell-surface receptors, such as RAS, or receptor tyrosine kinases, such as FLT3 and c-KIT. Through various downstream pathways, constitutive activation confers a survival or proliferative advantage leading to clonal expansion of the affected hematopoietic progenitors. In mouse models, abnormalities in RAS, FLT3 or c-KIT by themselves produce only a myeloproliferative disorder and not AML. The second type of lesion (class 2) exemplified by overexpression of HOX genes or formation of fusion genes, resulting from the $t(8 ; 21)$ or inv(16) abnormalities block myeloid differentiation but, similar to class 1 lesions, do not cause leukemia in mouse models. AML may develop only when both classes of lesions are present (2-7). Current efforts in clinical research have focused on the assessment of targeted therapies. Such new approaches may lead to an increase in the cure rate. At present, the strategy of gene therapy may offer new hope for expanded treatment options for patients with AML.

To improve therapeutic effect, the gene therapy treatment strategy has been suggested as a future direction. Gene therapy has emerged as a powerful tool with which to regulate biological functions in diseased tissues and to treat cancers. Oncolytic viruses not only have the capacity to express therapeutic genes in tumor cells, but also can be used as a direct tumor-destruction medicament. For safety, oncolytic viral replication must be controlled strictly within tumor cells (8-11). Thus, different types of viruses have been genetically modified, including adenovirus, adeno-associated virus (AAV), herpes simplex virus type I, reovirus, lentivirus, hepatitis B virus and Newcastle disease virus (12-16). One of the common strategies used to design oncolytic adenoviruses is to modify adenoviral E1A protein. The CR2 region of the adenoviral E1A binds to retinoblastoma protein $(\mathrm{RB})$ and the RB-related proteins which regulate the $\mathrm{E} 2 \mathrm{~F}$ family of transcription factors and induces quiescent cells to enter the $\mathrm{S}$ phase. Since tumor cells often have dysfunctional RB and an uncontrolled cell cycle, deletion of the CR 2 region allows this engineered adenovirus to selectively replicate in tumor cells but not in quiescent normal cells. We previously constructed several 
conditionally replicative adenovirus systems in which viral replication only occurred in cancer cells with high expression of hTERT and an abnormal cell cycle checkpoint. However, among these oncolytic adenoviruses, therapeutic genes were controlled by exogenous constitutive promoters. Thus, expression of therapeutic genes in normal tissue may induce an undesired effect even if the virus does not replicate (17-21). To overcome this limitation, we developed the AdCN205 system in which therapeutic gene expression is controlled by the adenovirus E3 endogenous promoter. We confirmed that this vector expresses the therapeutic gene in a predictable and safe manner (22). The human adenovirus serotype 11 (Ad11), with a fiber different from that of Ad5, can enter cells which have a capacity for secreting complement regulatory protein CD46 (a specific membrane protein) to the cytomembrane. The Ad11 adenoviral vector is an alternative means for cancer therapy, particularly in leukemia. Therefore, we developed the AdCN205-11 system to selectively replicate in AML cell lines, and it exhibited marked antitumor activity. Chimeric oncolytic adenoviruses, which are increasingly being developed as oncolytic vectors, have recently emerged as a novel approach for treating various neoplasms (23-25). Because of their capability to replicate in transduced tumor cells but not in normal tissue, they infect neighboring tumor cells after selective viral propagation resulting in virus-mediated lysis of tumor cells and tumor regression. Chimeric oncolytic adenoviruses may have better antitumor efficacy than that of nonreplicating adenoviruses.

We chose melanoma differentiation-associated gene-7/interleukin-24 (MDA-7/IL-24), a novel member of the IL-10 family of cytokines, as inserted therapeutic genes to augment the anticancer effects of the AdCN205-11 system since MDA-7/IL-24 is a novel tumor-suppressor gene that plays an important role in tumorigenesis. The overexpression of MDA-7/IL-24 using a recombinant adenovirus causes in vitro selective growth suppression and apoptosis of a variety of tumor cells, including melanoma, glioblastoma multilforme, osteosarcoma and carcinomas of the breast, colon, lung, cervix, kidney and prostate (26-31). Studies by several laboratories have uncovered many of the unique properties of MDA-7/IL-24. These include cancer-specific apoptosis induction, cell cycle regulation, an ability to inhibit angiogenesis, potent 'bystander antitumor activity' and a capacity to enhance the sensitivity of tumor cells to radiation, chemotherapy and monoclonal antibody therapy. Moreover, based on its profound cancer tropism, substantiated by in vivo human xenograft studies in nude mice, MDA-7/IL-24 (administered as Ad.mda-7) was evaluated in a phase I clinical trial in patients with melanomas and solid cancers. These studies document that MDA-7/IL-24 is well tolerated and demonstrate the evidence of significant clinical activity. In these contexts, MDA-7/IL-24 represents a unique cytokine gene with the potential for therapy of human cancers. There are three unique properties of MDA-7/IL-24, namely its potent 'bystander antitumor activity', ability to sensitize tumor cells to radiation and its antiangiogenesis properties. Additionally, the phase I clinical trial is provided. These studies confirm that MDA-7/IL-24 has promise for the management of diverse types of cancers (26-31). In the present study, we confimed that the application of IL-24 in the gene therapy system has a dramatic antitumor effect.

\section{Materials and methods}

Cell cultures. The normal human liver L02 cell line was purchased from the Shanghai Cell Collection (Shanghai, China). The HEK293 cell line was purchased from Microbix Biosystems Inc. (Toronto, ON, Canada). The human AML KG-1 cell line was purchased from the American Type Culture Collection (ATCC; Rockville, MD, USA). L02 and HEK293 cells were maintained in Dulbecco's modified Eagle's medium (DMEM; Gibco-BRL, Grand Island, NY, USA) supplemented with $10 \%$ fetal bovine serum (FBS; Gibco-BRL), 4 mM glutamine, $50 \mathrm{U} / \mathrm{ml}$ penicillin and $50 \mathrm{mg} / \mathrm{ml}$ streptomycin. KG-1 cells were grown in RPMI-1640 medium containing $10 \%$ FBS. All cell lines were incubated at $37^{\circ} \mathrm{C}$ in a humidified air atmosphere with $5 \% \mathrm{CO}_{2}$.

Construction of the oncolytic adenoviral vectors. The constructs including pCN205-11-EGFP and pCN205-11-IL-24 were generated according to the standard molecular cloning protocol. The homologous recombination between the pCN205-11-EGFP and pCN205-11-IL-24 plasmids and the pCN103 plasmid carrying the oncolytic adenoviral backbone was carried out in E. coli strain BJ5183, to create pAdCN205EGFP and pAdCN205-IL-24, respectively. Viral particles were produced in HEK293 cells by transfection with $\mathrm{PacI}-$ digested pAdCN205-EGFP and pAdCN205-IL-24 to obtain recombinant AdCN205-11-EGFP and AdCN205-11-IL-24.

Generation, purification and titration of the recombinant adenoviruses. To obtain the viruses, the plasmids pCN20511-EGFP and pCN205-11-IL-24 were digested by PacI and transfected into HEK293 cells using Effectene (Qiagen, Hilden, Germany). The recombinant adenoviruses were amplified in HEK293 cells and purified by cesium chloride gradient ultracentrifugation. The titration of the recombinant adenovirus was carried out with Tissue Culture Infectious Dose (TCID) $)_{50}$ assay in HEK293 cells.

Virus progeny assay. To determine the virus progeny, acute myeloid leukemia or normal cells were infected with Ad-wt, AdCN205-EGFP, AdCN205-IL-24, AdCN205-11-EGFP or AdCN205-11-IL-24 at an MOI of 10. After $48 \mathrm{~h}$, the supernatants and cells were collected separately. The cells were resuspended in PBS, and the virus was released by three freeze-thaw cycles. Virus production in the supernatants and cell lysates was determined by TCID $_{50}$ assay in HEK293 cells.

Cell viability assay. Cells were seeded in 96-well plates at a density of $1 \times 10^{4}$ perwell. When cells grew to subconfluency, they were infected with Ad-wt, AdCN205-EGFP, AdCN205-IL-24, AdCN205-11-EGFP or AdCN205-11-IL-24 at a multiplicity of infection (MOI) of 10 . Fresh medium containing $0.5 \mathrm{mg} / \mathrm{ml}$ 3-(4,5-dimethylthiazol-2-yl)-2,5-diphenyltetrazolium bromide (MTT) (Sigma Chemical Co., St. Louis, MO, USA) solution was added to each well at different times after infection. Cells were incubated at $37^{\circ} \mathrm{C}$ for $4 \mathrm{~h}$ and then $150 \mu \mathrm{l}$ of dimethyl sulfoxide was added to each well and mixed thoroughly for $10 \mathrm{~min}$. Absorbance was read at $595 \mathrm{~nm}$ with a DNA Expert (Tecan, Switzerland). 
A
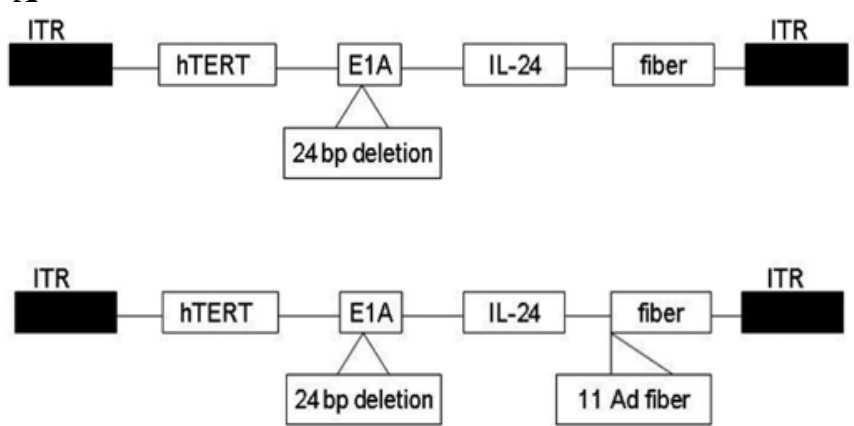

B

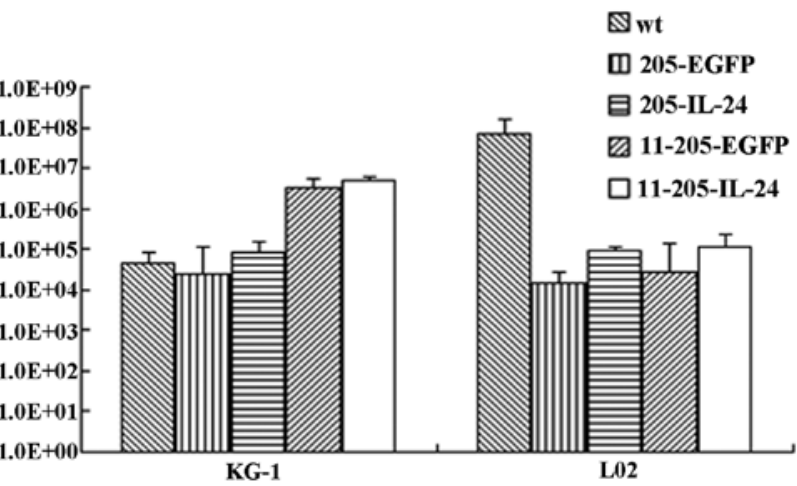

Figure 1. Construction and characterization of the oncolytic adenoviral vectors. (A) Structures of the oncolytic adenoviral vectors carrying EGFP and IL-24 Schematic description of the structures of AdCN205, AdCN205-11-GFP and AdCN205-11-IL24. In AdCN205, the E1A promoter was replaced by the hTERT promoter and deletion of the adenoviral genome 923 to 946 nucleotides, which enables viral replication within malignant cells with abnormal RB function. The fibers on the human adenovirus type 5 (Ad5) were replaced by fibers on the human adenovirus type 11 (Ad11). In AdCN205-11-GFP and AdCN205-11-IL-24, E3 6.7K/gp19K genes were substituted by the GFP reporter gene and the hIL-24 therapeutic gene, respectively. (B) Selective replication of the recombinant adenoviruses in vitro. The AML KG-1 and normal L02 cell lines were infected with Ad-wt, AdCN205-EGFP, AdCN205-IL-24, AdCN205-11-EGFP or AdCN205-11-IL-24 at an MOI of 10. After $48 \mathrm{~h}$, the supernatants and cells were collected separately. The cells were resuspended in PBS and subjected to three freeze-thaw cycles. The virus yield was measured in the supernatants and cell lysates. "P<0.01 as compared with the AdCN205-EGFP or Ad-wt group. IL-24, interleukin-24; AML, acute myeloid leukemia; MOI, multiplicity of infection.

In vitro transduction studies. For suspension of cells, the day before infection, $3 \times 10^{5}$ cells per well (24-well plate) were seeded. The next day, the attached cells were counted and the viruses were added at the multiplicities of infection (MOIs) indicated in the figure legends in $1 \mathrm{ml}$ of growth medium. Cells were incubated with the virus for $24-48 \mathrm{~h}$, and incubated at $37^{\circ} \mathrm{C}$ in a humidified atmosphere with $5 \% \mathrm{CO}_{2}$. Percentages of GFP-positive cells were detected by fluorescence microscopy.

Hoechst 33342 staining and apoptotic cell staining. Cells $\left(4 \times 10^{5}\right)$ were cultured in each well of 6-well plates. Twelve hours later, the cells were infected with Ad-wt, AdCN205-EGFP, AdCN205-IL-24, AdCN205-11-EGFP or AdCN205-11-IL-24 at an MOI of 10. Forty-eight hours after infection, the medium was replaced with PBS and then the cells were stained with Hoechst $33342(25 \mu \mathrm{g} / \mathrm{ml})$. The percentage of apoptotic cells was analyzed with and observed under a fluorescence microscope.

Flow cytometric analysis. For apoptosis detection, the $\mathrm{KG}-1$ cells were seeded in 6-well culture plates and infected with Ad-wt, AdCN205-EGFP, AdCN205-IL-24, AdCN205-11-EGFP or AdCN205-11-IL24 at an MOI of 10. Cells infected with the adenovirus were trypsinized and washed once with complete medium. Aliquots of cells $\left(5 \times 10^{5}\right)$ were resuspended in $500 \mu \mathrm{l}$ of binding buffer and stained with fluorescein isothiocyanate-labeled Annexin $\mathrm{V}$ and propidium iodide (PI; BioVision, Palo Alto, CA, USA) according to the manufacturer's instructions. A fluorescence-activated cell sorting (BD Biosciences) assay was performed immediately after staining. To determine transduction efficiency of the virus, KG-1 cells were infected with Ad-wt, AdCN205-IL-24 or AdCN205-11-IL-24 at an MOI of 10 for $48 \mathrm{~h}$ and then subjected to flow cytometric analysis.

Western blot analysis. To determine the expression of various proteins, western blot analysis was performed as described previously. The cells were harvested by trypsinization and resuspended in lysis buffer [62.5 mM Tris- $\mathrm{HCl}(\mathrm{pH} 6.8), 2 \%$ sodium dodecylsulfate, $10 \mathrm{mM}$ glycerol and $1.55 \%$ dithiothreitol]. The total protein concentration was determined by the BCA protein assay kit (Pierce Corporation, Rockford, IL, USA). The protein samples were separated by $10 \%$ sodium dodecylsulfate-polyacrylamide gel electrophoresis and transferred to nitrocellulose membranes (Millipore Corporation, Billerica, MA, USA). The membranes were blocked in a $5 \%$ bovine serum albumin solution and incubated with the primary antibodies, followed by secondary fluorescent antibodies. The primary antibodies included rabbit monoclonal anti-mda-7/IL-24 (Abcam, Cambridge, UK), rabbit polyclonal anti-cleaved caspase-3 (Cell Signaling Technology, Danvers, MA, USA), poly(ADP-ribose) polymerase-1/2 (H-250) rabbit polyclonal antibody (Santa Cruz Biotechnology, Santa Cruz, CA, USA) and $\beta$-actin, rabbit monoclonal (Epitomics, Burlingame, CA, USA). The membranes were then incubated with anti-rabbit infrared dye 700. The fluorescent signal was detected with an Odyssey Infrared Imaging System (LI-COR Biosciences, Lincoln, NE, USA).

Statistical analysis. The data reported represent the means of three independent experiments, and the bars indicate the standard deviation. The Student's t-test was used to calculate the statistical significance of the experimental results. The level of significance was set at $\mathrm{P}<0.05$.

\section{Results}

The construction and characterization of the Ad5/11 chimeric oncolytic adenoviruses. Previously, our group developed a double-controlled oncolytic adenovirus system, AdCN205, in which the hTERT promoter was used to control the expression of the CR2-deleted E1A region, and the $6.7 \mathrm{~K} / \mathrm{gp} 19 \mathrm{~K}$ of E3 region were substituted by the exogenous genes. This vector allows selective adenoviral replication in tumor cells harboring overexpression of hTERT and dysfunction of RB. The exogenous genes in the vector controlled by the adeno- 


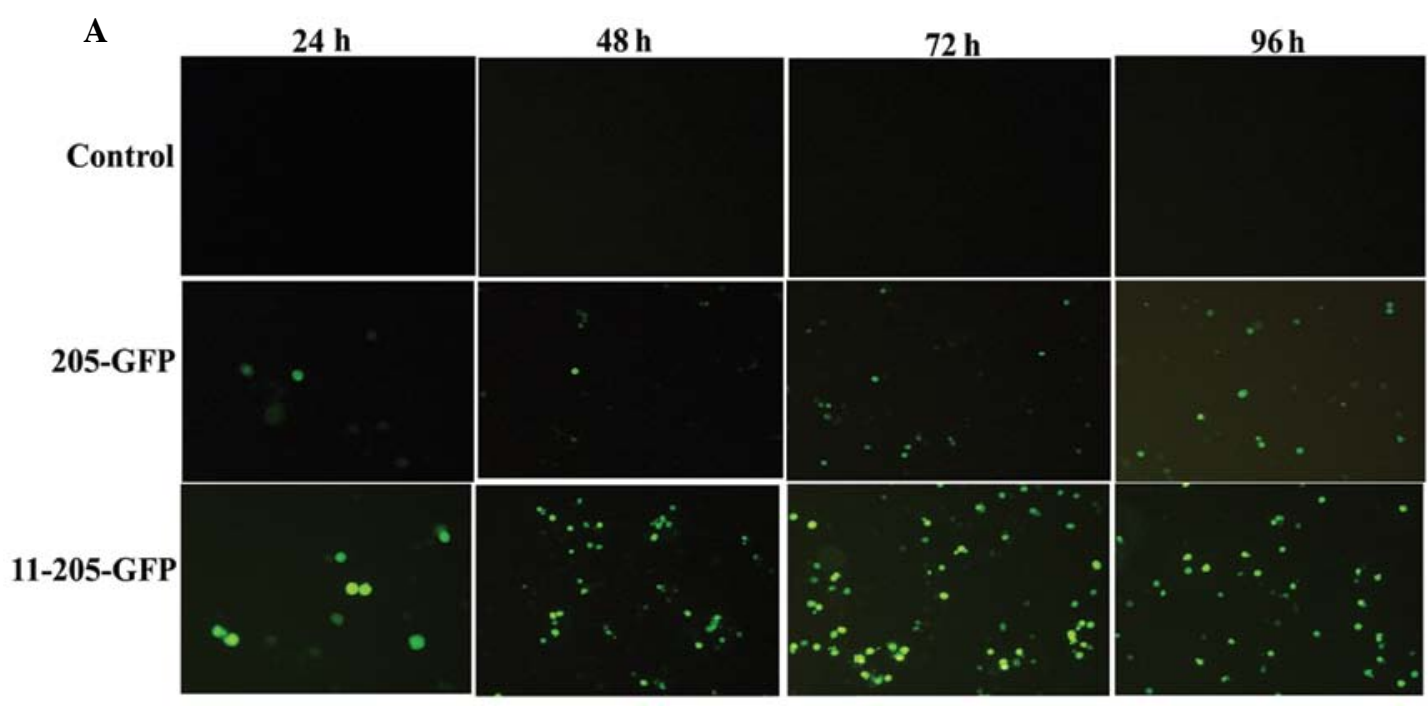

$\mathbf{B}$
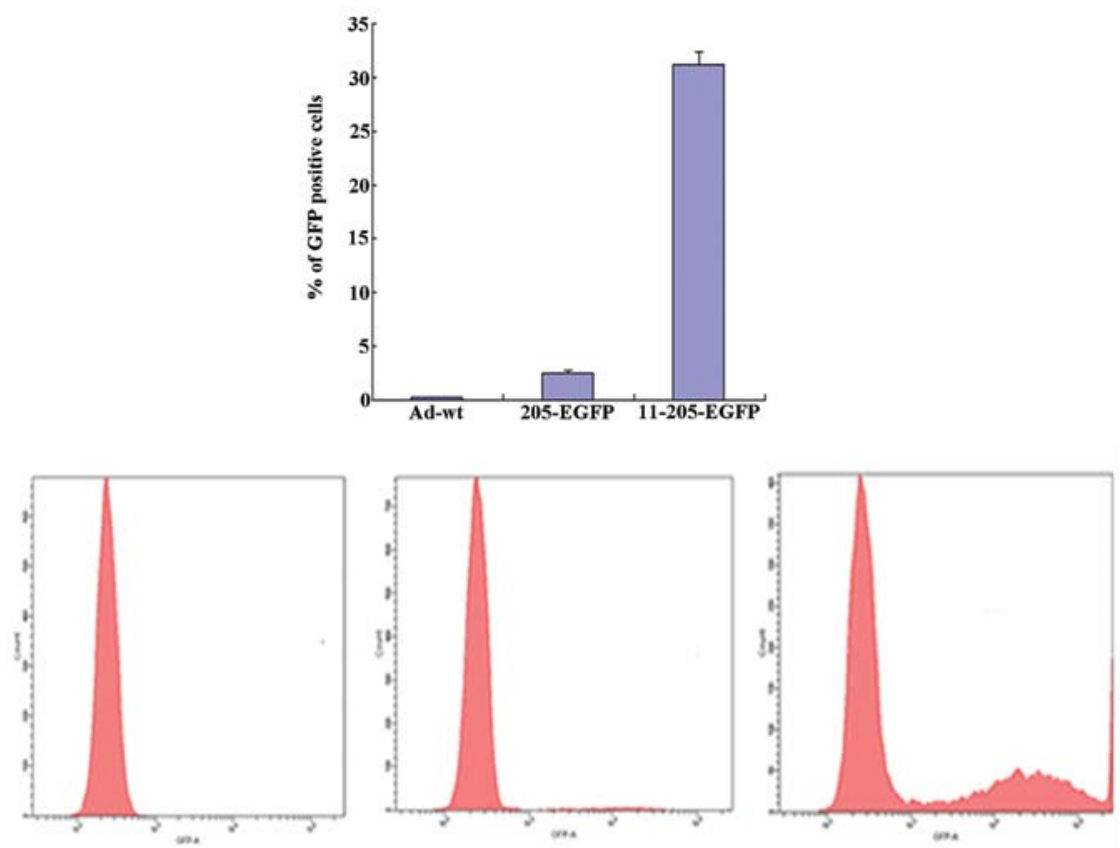

C

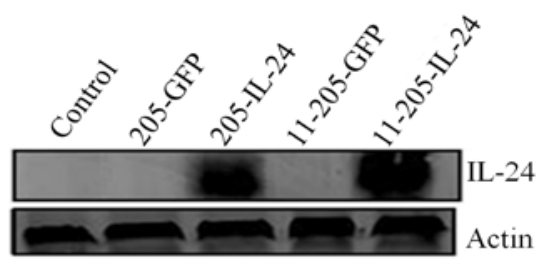

Figure 2. Transduction efficiency by recombinant adenoviruses in vitro. Representative photomicrographs were obtained from KG-1 cells infected with AdCN205-EGFP, AdCN205-11-EGFP or Ad-wt at an MOI of 10. Original magnification, x200. (B) The transduction efficiency of the virus. KG-1 cells were infected with Ad-wt, AdCN205-EGFP or AdCN205-11-EGFP at an MOI of 10 for $48 \mathrm{~h}$ and then subjected to flow cytometric analysis. (C) Detection of IL-24 in KG-1 cells after infection with Ad-wt, AdCN205-EGFP, AdCN205-IL-24, AdCN205-11-EGFP or AdCN205-11-IL-24 at an MOI of 10. The total protein from the treated cells after $48 \mathrm{~h}$ of infection was subjected to western blot analysis with antibodies against IL-24 and $\beta$-actin. IL-24, interleukin-24; AML, acute myeloid leukemia; MOI, multiplicity of infection.

virus endogenous E3 promoter are expressed in tumor cells following virus replication. In the present study, we engineered the Ad5/11 chimeric oncolytic adenovirus AdCN205-11-EGFP or AdCN205-11-IL-24 by replacement of the Ad5 fiber from the AdCN205 system with the Ad11 fiber. The structures of the Ad5/11 chimeric oncolytic adenovirus AdCN205-11-GFP and AdCN205-11-IL-24 are shown in Fig. 1A.
Selective replication of the oncolytic adenoviral vectors in vitro. To examine whether the transgenes could interfere with the selective replication ability of the recombinant adenoviruses, a progeny assay was conducted in the AML cells (KG-1) and normal cells (L02) infected with Ad-wt, AdCN205-EGFP, AdCN205-IL-24, AdCN205-11-EGFP or AdCN205-11-IL-24 at an MOI of 10. As shown in Fig. 1B, 


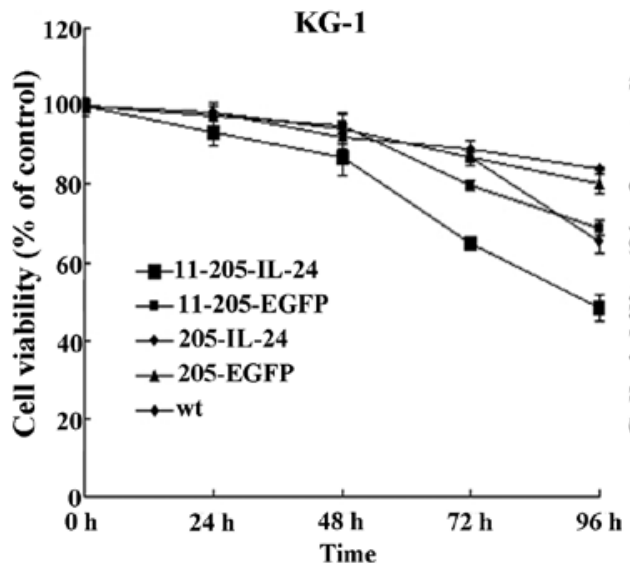

B

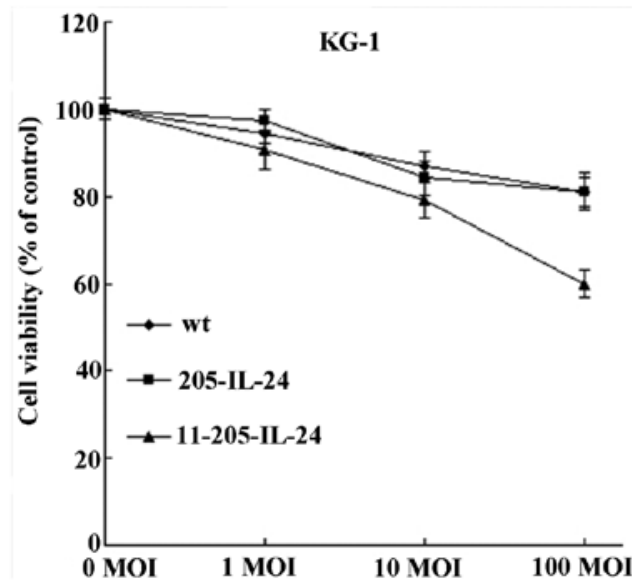

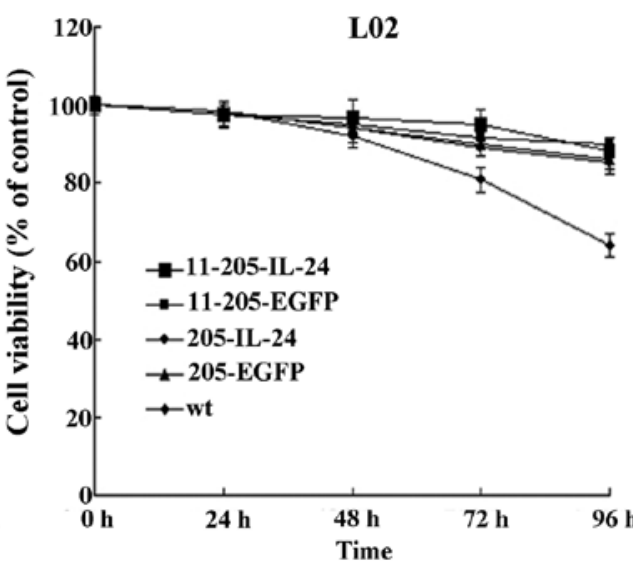

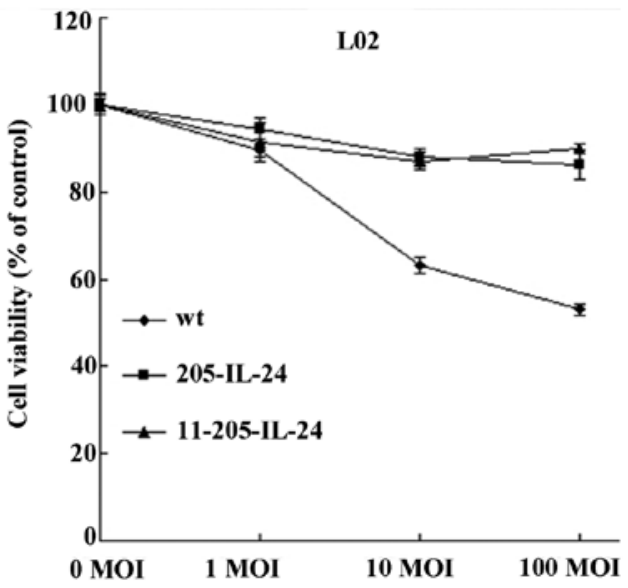

Figure 3. Induction of cytotoxicity by the oncolytic adenoviral vectors in vitro. (A). The AML KG-1 and normal L02 cell lines were infected with Ad-wt, AdCN205-EGFP, AdCN205-IL-24, AdCN205-11-EGFP or AdCN205-11-IL-24 at an MOI of 10. Cell viability was determined by the MTT assay at 24, 48, 72 and 96 h after infection. (B) KG-1 and L02 cells were infected with Ad-wt, AdCN205-IL-24 or AdCN205-11-IL-24 at different MOIs. Cell viability was determined by the MTT assay at $96 \mathrm{~h}$ after infection. The cell viability was calculated as a percentage with respect to the cells without viral infection. Data are presented as the means \pm SD of three independent experiments. ${ }^{*} \mathrm{P}<0.01$ as compared with the AdCN205-EGFP or Ad-wt group. AML, acute myeloid leukemia; MOI, multiplicity of infection.

AdCN205-11-IL-24 and AdCN205-11-EGFP replicated at similar levels in the AML cells; these levels were higher than the levels in AML cells infected with Ad-wt, AdCN205-EGFP or AdCN205-IL-24. In contrast, the replication capacity of these vectors was markedly reduced in the normal cells. These data indicate that expression of IL-24 and EGFP did not affect the selective replication ability of the oncolytic vectors.

Furthermore, to confirm whether the exogenous gene could be expressed properly in the AML cells infected with the oncolytic adenoviruses, the KG-1 cell line was infected with AdCN205-EGFP, AdCN205-11-EGFP or Ad-wt at an MOI of 10. Our results showed that the KG-1 cells infected with AdCN205-11-EGFP expressed a high level of EGFP protein as compared with the KG-1 cells infected with AdCN205-EGFP and Ad-wt as observed under fluorescence microscopy (Fig. 2A). To evaluted the transduction efficiency of the recombinant oncolytic adenoviral, KG-1 cells were infected with Ad-wt, AdCN205-EGFP or AdCN205-11-EGFP at an MOI of 10 for $48 \mathrm{~h}$ and then subjected to flow cytometric analysis. AdCN20511-EGFP transduced KG-1 cells with high efficiency (Fig. 2B). Similarly, a high level of IL-24 was observed in the KG-1 cells after infection with AdCN205-11-IL-24 (Fig. 2C). These data indicate that the Ad5/11 chimeric oncolytic adenovirus could selectively replicate in the AML cells and express a high level of the transgenes.

A cytotoxic effect is induced by the oncolytic adenoviral vectors in AML cells in vitro. To analyze the antitumor efficiency of the recombinant adenoviruses, the AML cell line (KG-1) and a normal cell line (L02) were infected with Ad-wt, AdCN205-EGFP, AdCN205-IL-24, AdCN205-11-EGFP or AdCN205-11-IL-24 at an MOI of 10, and cell survival was determined by the MTT assay. As shown in Fig. 3A, the AdCN205-EGFP, AdCN205-IL-24 and Ad-wt induced a cytotoxic effect on AML cells at a similar level in a time-dependent manner. Moreover, AdCN205-11-IL-24 significantly reduced the viability of the AML cells, as compared with that induced by Ad-wt, AdCN205-EGFP, AdCN205-IL-24 or AdCN205-11EGFP. In contrast, oncolytic adenoviruses AdCN205-11-EGFP and AdCN205-11-IL-24 did not induce a cytotoxic effect on the normal cell line L02. Furthermore, we observed that AdCN205-11-IL-24 increased the cytotoxicity to AML cells in a dose-dependent manner, as compared with that induced by AdCN205-IL-24 and Ad-wt (Fig. 3B). These data indicate that AdCN205-11-IL-24 can exert an obvious cytotoxic effect on AML cells in vitro. 

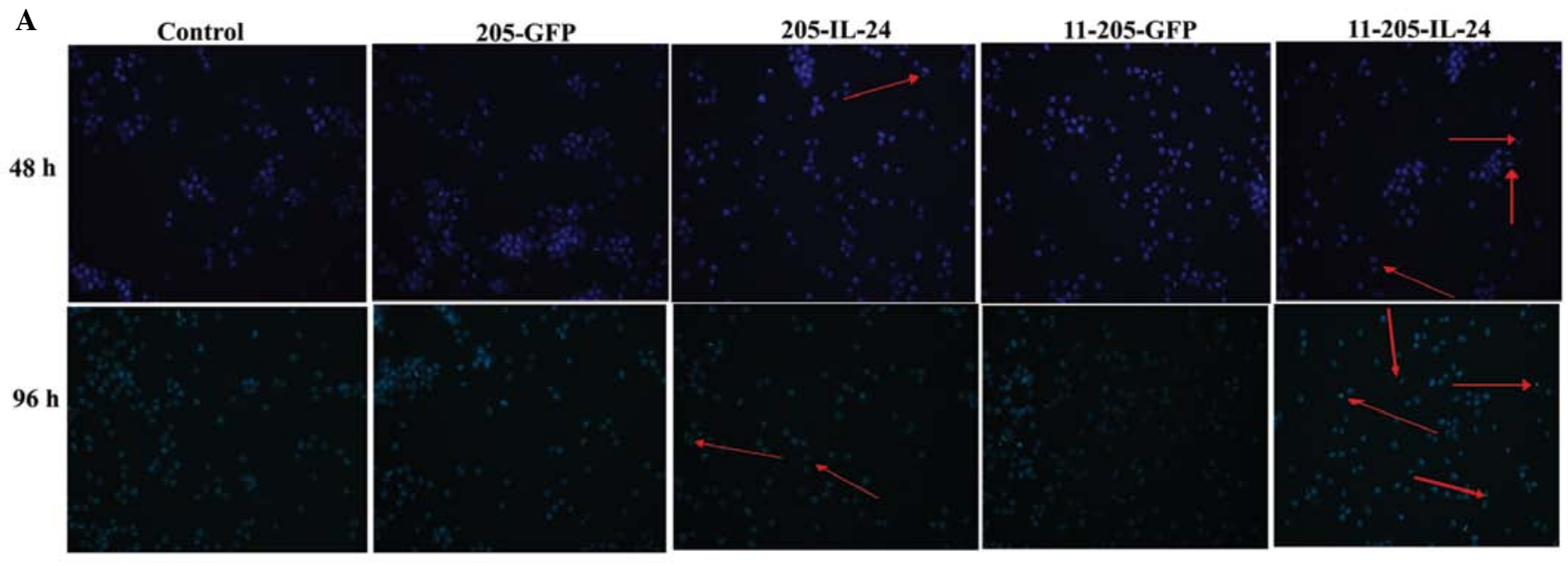

B
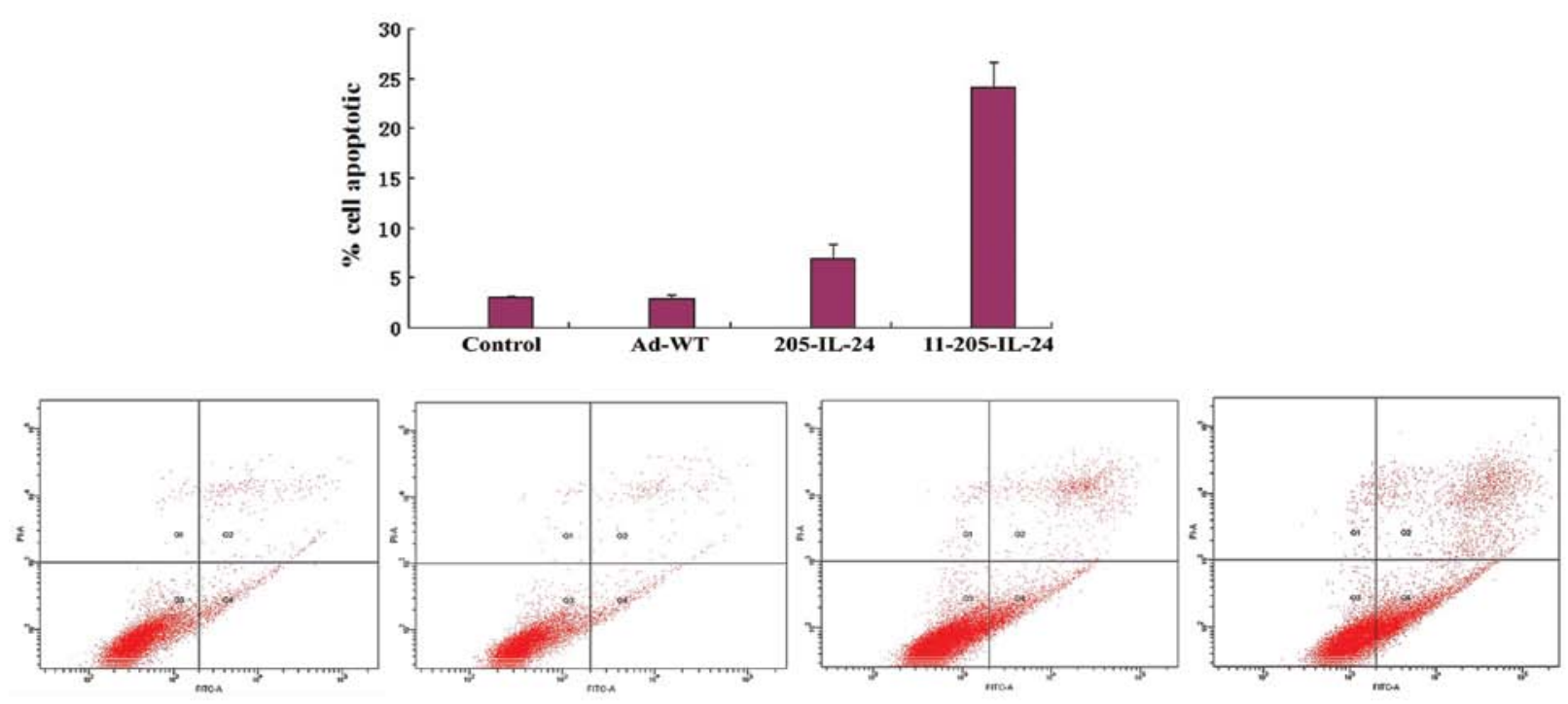

C

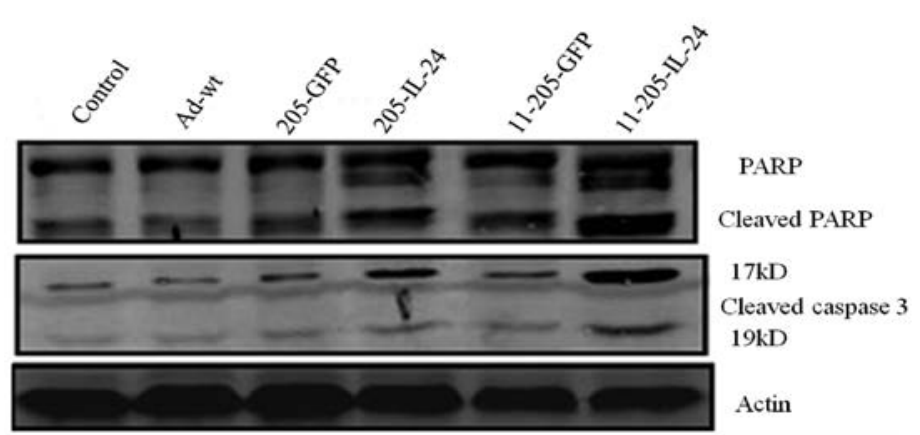

Figure 4. Induction of cell apoptosis in AML cells after infection with AdCN205-11-IL-24 in vitro. (A) AML cells were stained with Hoechst 33342 after infection with Ad-wt, AdCN205-EGFP, AdCN205-IL-24, AdCN205-11-EGFP or AdCN205-11-IL-24 at an MOI of 10 for 48 h. Arrows indicate positive apoptotic cells. Original magnification, x100. (B) Detection of apoptosis by staining with anti-Annexin V in KG-1 cells after infection with Ad-wt, AdCN205-IL-24 or AdCN205-11-IL-24 at an MOI of 10, after $48 \mathrm{~h}$ by flow cytometric analysis. (C) Detection of cleaved caspase 3 and PARP in KG-1 cells after infection with Ad-wt, AdCN205-EGFP, AdCN205-IL-24, AdCN205-11-EGFP or AdCN205-11-IL-24 at an MOI of 10. The total protein from the treated cells after 48 h of infection was subjected to western blot analysis with antibodies against total IL-24, cleaved caspase 3, PARP and $\beta$-actin. AML, acute myeloid leukemia; MOI, multiplicity of infection; IL-24, interleukin-24.

Induction of apoptosis in AML cells after infection with the oncolytic adenoviral vectors in vitro. To examine whether IL-24 induces apoptosis in AML cells, AML cells were infected with Ad-wt, AdCN205-EGFP, AdCN205-IL-24, AdCN205-11-EGFP or AdCN205-11-IL-24 at an MOI of 10 for 48 h. Fig. 4 A shows that infection of KG-1 cells with AdCN205-
11-IL-24 led to marked morphological changes characteristic of apoptosis, such as chromatin condensation, nuclear fragmentation and apoptotic body formation in the KG-1 cells. Fluorescence-activated cell sorting analysis was performed to examine apoptosis induced by AdCN205-11-IL-24. Our results showed that the apoptotic rate induced by AdCN205-11-IL-24 
was significantly higher than that induced by AdCN205-EGFP, AdCN205-IL-24 or Ad-wt (Fig. 4B). Furthermore, activation of the caspase pathway was examined to explore the mechanism of AML cell apoptosis. As shown in Fig. 4C, the cleavage of caspase 3 and PARP was obviously increased in the KG-1 cells treated with AdCN205-11-IL-24, as compared with Ad-wt, AdCN205-EGFP, AdCN205-IL-24, AdCN-EGFP or phosphate-buffered saline (PBS). These results suggest that AdCN205-11-IL-24 markedly induced KG-1 cell apoptosis by activation of extrinsic pathways.

\section{Discussion}

Human leukemias are characterized by acquired recurring chromosomal translocations. Cloning of these translocation breakpoints has provided important insight into the pathogenesis of the disease as well as novel therapeutic approaches. Acute myeloid leukemia (AML) is characterized by uncontrolled proliferation, increased survival and impaired differentiation of hematopoietic progenitor cells. Analysis of primary human AML samples and data from animal models of leukemia have demonstrated that these characteristics can be attributed to deregulation of signal transduction pathways and disruption of cellular differentiation programs that cooperate in AML pathogenesis. These activated kinases are validated targets for therapy with selective tyrosine kinase inhibitors, a paradigm that may have broad applications in the treatment of hematologic malignancies as well as solid tumors. This represents a critical issue in the effort to design molecular therapies.

Activation of signal transduction in AML may occur through a variety of genetic alterations affecting different signaling molecules, such as the FLT3 and KIT receptor tyrosine kinases (RTKs) and members of the RAS family of guanine nucleotide-binding proteins. These mutant signaling proteins are attractive therapeutic targets; however, the development of targeted therapies for each genotypic variant and determining the relationships between different genotypes and critical functional dependencies of leukemic cells remain major challenges. As the large number of mutant signaling proteins that have been identified in AML are likely to reflect activation of a more limited number of downstream effector pathways, such as the RAF/MEK/ERK and PI3K/AKT cascades, targeting these unifying pathways may represent a more broadly applicable therapeutic strategy. Furthermore, integrative genomic studies combining DNA sequencing, DNA copy number analysis, transcriptional profiling and functional genetic approaches hold great promise for identifying additional signaling abnormalities in AML that are relevant to leukemogenesis and can be exploited therapeutically. Eventually, it may become possible to use pathogenesis-oriented combinations of signal transduction inhibitors to improve the cure rate in AML patients. Biological therapies, including gene therapy, have shown great promise for the treatment of AML. However, present strategies are not effective enough to eliminate cancer completely, particularly in human patients. Therefore, investigation of potent vectors and therapeutic genes for this purpose are needed.

Research has uncovered a specific gene, melanoma differentiation-associated gene-7/interleukin-24 (MDA-7/IL-24), displaying cancer-specific apoptosis-inducing properties.
Isolation using this scheme has now come into the limelight as a new gene therapy for divergent types of cancers. Although the mechanism of cancer cell selectivity of MDA-7/IL-24 remains to be verified, numerous attributes enable this gene to be an effective therapy for cancer, including the ability to discriminate between normal and cancer cells, to induce apoptosis in diverse tumor cells, to promote 'bystander' antitumor effects, to inhibit tumor growth and angiogenesis in animal models, to synergize with radiation and to modulate immune responses. These unique features combined with successful transition into the clinic provides hope that IL-24, as a single or more likely as part of a combinatorial approach, may provide profound therapeutic benefit for cancer patients. Our previous study confirmed that IL-24 exerted strong antitumor activity in vitro and in vivo. A clinical trial with a replication-defective adenovirus expressing IL-24 has shown a good safety profile and some partial responses in patients with cancers. In the present study, we aimed to ascertan whether the 'magical bullet' IL-24 could be used as a therapeutic gene for the treatment of AML.

To achieve the maximal transduction efficiency in cancer cells, application of the replication-competent viral vectors is required. It has been shown that oncolytic adenoviruses transduce tumor cells effectively and exhibit an adequate safety profile. In our previous studies, we constructed the doubleregulated oncolytic adenoviral vectors (AdCN205-EGFP and AdCN205-IL-24) that could target human telomerase reverse transcriptase and retinoblastoma pathways $(18,32,33)$. At the same time, we constructed oncolytic adenoviral vectors in which fibers on the human adenovirus type 5 (Ad5) were replaced with the human adenovirus type 11 (Ad11) that can only target specific selective replication of recombinant adenoviruses in leukemia cells. This vector only selectively replicates in AML cells $(34,35)$. Therefore, we used this vector to deliver the IL-24 gene (AdCN205-11-EGFP and AdCN20511-IL-24) for the therapy of AML.

Our data revealed that AdCN205-11-IL-24 exhibited a strong cytotoxic effect on AML cells and this cytotoxic effect was much higher than that induced by Ad-wt, AdCN205-EGFP or AdCN205-IL-24. Most importantly, AdCN205-11-IL-24 could not replicate in normal cells and it did not induce any cytotoxicity in normal cells. We also demonstrated that transfer of IL-24 by AdCN205-11-IL-24 resulted in marked expression in AML cells, and AdCN205-IL-24 was barely detected. These results indicate that the strong cytotoxic effect of AdCN205-11-IL-24 in AML cells was caused by alteration of the cell cycle and induction of cell apoptosis. Our data further demonstrated that AdCN205-11-IL-24 induced cell apoptosis by induction of the cleavage of caspase 3 and PARP, the main and important mechanisms for induction of cell apoptosis. The present study revealed that AdCN205-11-IL-24 induced AML cell apoptosis through the activation of the extrinsic pathway.

Taken together, AdCN205-11-IL-24, a chimeric oncolytic adenovirus harboring IL-24, was successfully generated. This vector selectively replicates in AML cells and exhibits marked antitumor activity in vitro. Restoration of IL-24 by chimeric oncolytic adenovirus could induce AML cell apoptosis through the activation of the extrinsic pathway in vitro. This study provides promise for the development of a novel gene therapeutic strategy for AML. 


\section{Acknowledgements}

We thank Dr Cheng Qian for his kind assistance.

\section{References}

1. Estey E and Dohner H: Acute myeloid leukaemia. Lancet 368: 1894-1907, 2006.

2. Frohling S, Scholl C, Gilliland DG and Levine RL: Genetics of myeloid malignancies: pathogenetic and clinical implications J Clin Oncol 23: 6285-6295, 2005.

3. Appelbaum FR, Gundacker H, Head DR, et al: Age and acute myeloid leukemia. Blood 107: 3481-3485, 2006.

4. Downing JR: The core-binding factor leukemias: lessons learned from murine models. Curr Opin Genet Dev 13: 48-54, 2003.

5. Scholl C, Gilliland DG and Frohling S: Deregulation of signaling pathways in acute myeloid leukemia. Semin Oncol 35: 336-345, 2008.

6. Kornblau SM, Womble M, Qiu YH, et al: Simultaneous activation of multiple signal transduction pathways confers poor prognosis in acute myelogenous leukemia. Blood 108: 2358-2365, 2006.

7. Steelman LS, Pohnert SC, Shelton JG, Franklin RA, Bertrand FE and McCubrey JA: JAK/STAT, Raf/MEK/ERK, PI3K/Akt and BCR-ABL in cell cycle progression and leukemogenesis. Leukemia 18: 189-218, 2004

8. Sangro B and Prieto J: Gene therapy for liver cancer: clinical experience and future prospects. Curr Opin Mol Ther 12: 561-569, 2010.

9. Denefle PP: Introduction to gene therapy: a clinical aftermath. Methods Mol Biol 737: 27-44, 2011.

10. Wadhwa PD, Zielske SP, Roth JC, Ballas CB, Bowman JE and Gerson SL: Cancer gene therapy: scientific basis. Annu Rev Med 53: 437-452, 2002.

11. Pfeifer A and Verma IM: Gene therapy: promises and problems. Annu Rev Genomics Hum Genet 2: 177-211, 2001.

12. Qian C, Sangro B and Prieto J: New strategies to enhance gene therapy efficiency. Gastroenterology 123: 639-642, 2002.

13. Wong HH, Lemoine NR and Wang Y: Oncolytic viruses for cancer therapy: overcoming the obstacles. Viruses 2: 78-106, 2010.

14. Qian C, Liu XY and Prieto J: Therapy of cancer by cytokines mediated by gene therapy approach. Cell Res 16: 182-188, 2006.

15. Kirn D, Martuza RL and Zwiebel J: Replication-selective virotherapy for cancer: biological principles, risk management and future directions. Nat Med 7: 781-787, 2001.

16. Singer O and Verma IM: Applications of lentiviral vectors for shRNA delivery and transgenesis. Curr Gene Ther 8: 483-488, 2008.

17. Berk AJ: Recent lessons in gene expression, cell cycle control, and cell biology from adenovirus. Oncogene 24: 7673-7685, 2005.

18. Zhang W, Cai R, Luo J, et al: The oncolytic adenovirus targeting to TERT and RB pathway induced specific and potent anti-tumor efficacy in vitro and in vivo for hepatocellular carcinoma. Cancer Biol Ther 6: 1726-1732, 2007.
19. Sherr CJ and McCormick F: The RB and $\mathrm{p} 53$ pathways in cancer. Cancer Cell 2: 103-112, 2002.

20. Cui Q, Jiang W, Wang Y, et al: Transfer of suppressor of cytokine signaling 3 by an oncolytic adenovirus induces potential antitumor activities in hepatocellular carcinoma. Hepatology 47: 105-112, 2008

21. Papadakis ED, Nicklin SA, Baker AH and White SJ: Promoters and control elements: designing expression cassettes for gene therapy. Curr Gene Ther 4: 89-113, 2004.

22. Luo J, Xia Q, Zhang R, et al: Treatment of cancer with a novel dual-targeted conditionally replicative adenovirus armed with $m d a-7 / I L-24$ gene. Clin Cancer Res 14: 2450-2457, 2008.

23. Segerman A, Mei YF and Wadell G: Adenovirus types 11p and $35 p$ show high binding efficiencies for committed hematopoietic cell lines and are infective to these cell lines. J Virol 74: 1457-1467, 2000.

24. Lyons M, Onion D, Green NK, et al: Adenovirus type 5 interactions with human blood cells may compromise systemic delivery. Mol Ther 14: 118-128, 2006.

25. Yu L, Shimozato O, Li Q, et al: Adenovirus type 5 substituted with type 11 or 35 fiber structure increases its infectivity to human cells enabling dual gene transfer in CD46-dependent and -independent manners. Anticancer Res 27: 2311-2316, 2007.

26. Pestka S, Krause CD, Sarkar D, Walter MR, Shi Y and Fisher PB: Interleukin-10 and related cytokines and receptors. Annu Rev Immunol 22: 929-979, 2004.

27. Lebedeva IV, Emdad L, Su ZZ, et al: mda-7/IL-24, novel anticancer cytokine: Focus on bystander antitumor, radiosensitization and antiangiogenic properties and overview of the phase I clinical experience (Review). Int J Oncol 31: 985-1007, 2007.

28. Fisher PB: Is $m d a-7 / I L-24$ a 'magic bullet' for cancer? Cancer Res 65: 10128-10138, 2005.

29. Sauane M, Gopalkrishnan RV, Sarkar D, et al: MDA-7/IL-24: novel cancer growth suppressing and apoptosis inducing cytokine. Cytokine Growth Factor Rev 14: 35-51, 2003.

30. Qian W, Liu J, Tong Y, et al: Enhanced antitumor activity by a selective conditionally replicating adenovirus combining with MDA-7/interleukin-24 for B-lymphoblastic leukemia via induction of apoptosis. Leukemia 22: 361-369, 2008.

31. Zhao L, Dong A, Gu J, et al: The antitumor activity of TRAIL and IL-24 with replicating oncolytic adenovirus in colorectal cancer. Cancer Gene Ther 13: 1011-1022, 2006.

32. Aghi M and Martuza RL: Oncolytic viral therapies - the clinical experience. Oncogene 24: 7802-7816, 2005.

33. Mathis JM, Stoff-Khalili MA and Curiel DT: Oncolytic adenoviruses - selective retargeting to tumor cells. Oncogene 24: 7775-7791, 2005.

34. Wong HH, Jiang G, Gangeswaran R, et al: Modification of the early gene enhancer-promoter improves the oncolytic potency of adenovirus 11. Mol Ther 20: 306-316, 2012.

35. Segerman A, Atkinson JP, Marttila M, Dennerquist V, Wadell G and Arnberg N: Adenovirus type 11 uses CD46 as a cellular receptor. J Virol 77: 9183-9191, 2003. 